\title{
CONSTRUCTION OF SOME ALGEBRAS ASSOCIATED TO DIRECTED GRAPHS AND RELATED TO FACTORIZATIONS OF NONCOMMUTATIVE POLYNOMIALS
}

\author{
Vladimir Retakh ${ }^{1}$, Shirlei SerconeK ${ }^{2}$ and Robert Lee Wilson \\ AbStract. This is a survey of recently published results. We introduce and study a wide \\ class of algebras associated to directed graphs and related to factorizations of noncommutative \\ polynomials. In particular, we show that for many well-known graphs such algebras are Koszul \\ and compute their Hilbert series.
}

Let $R$ be an associative ring with unit and $P(t)=a_{0} t^{n}+a_{1} t^{n-1}+\cdots+a_{n}$ be a polynomial over $R$. Here $t$ is an independent central variable. We consider factorizations of $P(t)$ into a product

$$
P(t)=a_{0}\left(t-y_{n}\right)\left(t-y_{n-1}\right) \ldots\left(t-y_{1}\right)
$$

if such factorizations exist.

When $R$ is a (commutative) field, there is at most one such factorization up to a permutation of factors. When $R$ is not commutative, the polynomial $P(t)$ may have several essentially different factorizations.

The set of factorizations of a polynomial over a noncommutative ring can be rather complicated and studying them is a challenging and useful problem (see, for example, $[\mathrm{N}$, GLR, GR1, GR2, GRW, GGRW, LL, O, B, V, W]).

In this paper we present an approach relating such factorizations to algebras associated with directed graphs and study properties of such algebras.

In the factorization (0.1) the element $y_{1}$ is called a right root of $P(t)$ and element $y_{n}$ is called a left root of $P(t)$. This terminology can be justified by the following equalities (see, for example, $[\mathrm{L}])$ :

$$
a_{0} y_{1}^{n}+a_{1} y_{1}^{n-1}+\cdots+a_{n-1} y_{1}+a_{n}=0
$$

1991 Mathematics Subject Classification. 05E05; 15A15; 16W30.

Key words and phrases. noncommutative polynomials, directed graphs, Koszul algebras, Hilbert series. ${ }^{1}$ partially supported by NSA

${ }^{2}$ partially supported by $\mathrm{CNPq} / \mathrm{PADCT}$ 


$$
y_{n}^{n} a_{0}+y_{n}^{n-1} a_{1}+\cdots+y_{n} a_{n-1}+a_{n}=0 .
$$

It is natural (see [GGRSW]) to call the elements $y_{i}, i=1,2, \ldots, y_{n}$, in (0.1) pseudo-roots of $P(t)$. Note that any root is a pseudo-root but not every pseudo-root is a root.

There is a connection between factorizations of noncommutative polynomials and finding their right (left) roots and pseudo-roots. Consider the simplest non-trivial example when $P(t)=t^{2}+a_{1} t+a_{2}$ and $x_{1}, x_{2}$ are its right roots such that the difference $x_{1}-x_{2}$ is invertible in $R$.

Set

$$
\begin{aligned}
& x_{1,2}=\left(x_{2}-x_{1}\right) x_{2}\left(x_{2}-x_{1}\right)^{-1}, \\
& x_{2,1}=\left(x_{1}-x_{2}\right) x_{1}\left(x_{1}-x_{2}\right)^{-1} .
\end{aligned}
$$

One can show that

$$
P(t)=\left(t-x_{1,2}\right)\left(t-x_{1}\right)=\left(t-x_{2,1}\right)\left(t-x_{2}\right) .
$$

Thus we have two different factorizations of $P(t)$.

In studying factorizations of noncommutative polynomials, one should answer at least two questions:

1) How to obtain factorizations of type (0.1)?

2) How to relate different factorizations?

A partial answer to the first question was given in [GR1, GR2] when a polynomial $P(t)=t^{n}+a_{1} t^{n-1}+\cdots+a_{n}$ has $n$ right roots $x_{1}, x_{2}, \ldots, x_{n}$ in a generic position. Here elements $x_{1}, x_{2}, \ldots, x_{n}$ are in a generic position if all Vandermonde matrices $\left(x_{i_{\ell}}^{m-1}\right), \ell, m=$ $1,2, \ldots, k$ are invertible over $R$ when $k \geq 2$ and $i_{1}, i_{2}, \ldots, i_{k}$ are distinct. In [GR1, GR2] explicit formulas for $n$ ! factorizations of $P(t)$ were found. For $n=2$ these formulas give the factorizations $(0.2)$.

To answer the second question, one has to study relations among pseudo-roots of $P(t)$ and, more generally, study properties of subalgebras generated by pseudo-roots of $P(t)$.

For example, the factorizations (0.2) imply the following identities between the corresponding pseudo-roots:

$$
\begin{gathered}
x_{1,2}+x_{1}=x_{2,1}+x_{2}, \\
x_{1,2} x_{1}=x_{2,1} x_{2} .
\end{gathered}
$$

Note that one can consider expressions in (0.3) as noncommutative elementary symmetric functions of order 1 and 2 in $x_{1}, x_{2}$ (see [GR1, GR2, GRW, GGRW]). It is natural to study the algebra $Q_{2}$ with generators $x_{1,2}, x_{2,1}, x_{1}, x_{2}$ satisfying relations (0.3).

To carry out this idea, a universal algebra of $n$ pseudo-roots in a generic position, called $Q_{n}$, was introduced in [GRW] and its properties were studied in detail in [GRW, GGR, 
GGRSW, SW, Pi]. The algebras $Q_{n}$ are defined by linear and quadratic relations similar to relations (0.3). These algebras are Koszul and have nice Hilbert series. Overall, one can say that $Q_{n}$ is a rather "tame" algebra despite its exponential growth.

A more general approach to a study of algebras of pseudo-roots starts with $\Gamma_{P}$, a directed graph of right divisors of a polynomial $P(t)$ similar to the graph of divisors of a natural number. In this graph vertices correspond to right divisors of $P(t)$ and edges to pseudoroots of $P(t)$. Factorizations $(0.1)$ correspond to paths from a maximal vertex $P(t)$ to a minimal vertex 1 .

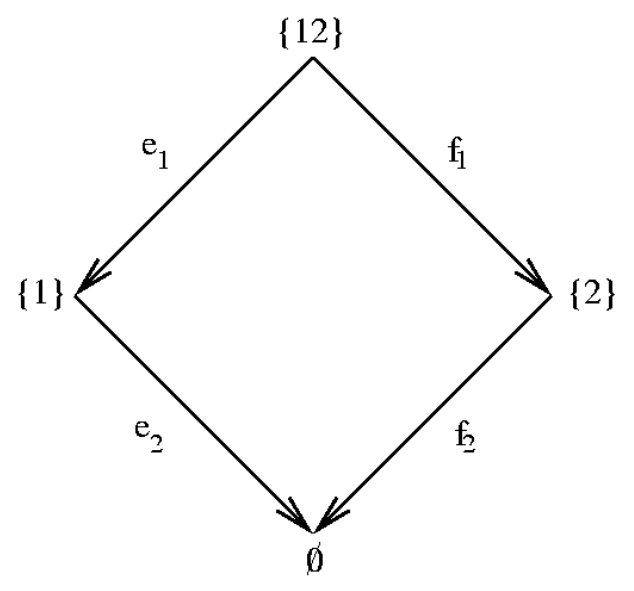

Figure 1

In this setting relations (0.3) for the algebra $Q_{2}$ can be described with a help of the diamond graph with vertices indexed by subsets of set $\{1,2\}$ (see Fig 1). Elements $x_{1,2}$ and $x_{2,1}$ correspond to edges $e_{1}$ and $f_{1}$, and elements $x_{1}$ and $x_{2}$ correspond to edges $e_{2}$ and $f_{2}$ respectively. Factorizations (0.2) correspond to two paths with the same origin and the end.

Our main objects are the "universal algebras" $A(\Gamma)$ associated to a directed graph $\Gamma$ and "universal polynomials" over these algebras. These algebras are generated by edges of $\Gamma$ and relations are defined by pairs of directed paths in $\Gamma$ with the same origin and end. If $\Gamma$ is the Hasse graph of the Boolean lattice of subsets of the set $\{1,2, \ldots, n\}$ then $A(\Gamma)=Q_{n}$.

It turns out that the algebras $A(\Gamma)$ have a lot of interesting properties similar to the algebras $Q_{n}$. The "geometric nature" of algebras $A(\Gamma)$ helps us to understand their structure and to simplify the proofs of main results for algebras $Q_{n}$ compared to the proofs given in [GRW, GGRSW, SW, Pi]. A geometric approach expresses coefficients of the "universal polynomials" via certain pseudo-roots of these polynomials, giving a geometric version of the noncommutative Viéte theorem from [GR1, GR2], see also [GGRW].

This paper contains definitions and results from [GRSW, RSW, RSW1, GGRW1]. It is organized in the following way. In Section 1 we recall the noncommutative Viéte theorem 
and the construction of the algebras $Q_{n}$. Section 2 contains a definition of the algebras $A(\Gamma)$ and similar algebras. In Section 3 we describe a linear basis in $A(\Gamma)$ and state that for a large class of directed graphs, the algebras $A(\Gamma)$ are Koszul. In Section 4 we compute Hilbert series for algebras $A(\Gamma)$ and discuss some examples. In Section 5 we describe a geometric version of the noncommutative Viéte theorem.

\section{The Viéte Theorem and algebras of PSEUDO-ROOTS OF NONCOMMUTATIVE POLYNOMIALS}

1.1. Factorizations of noncommutative polynomials. A generalization of the factorization (0.2) can be presented as follows (see [GR1, GR2, GRW]). Let $R$ be an associative ring with unit and $P(t)=t^{n}+a_{1} t^{n-1}+\cdots+a_{n}$ be polynomial in $R[t]$. Let $x_{1}, x_{2}, \ldots, x_{n} \in R$ be right roots of the polynomial, i.e. $x_{i}^{n}+a_{1} x_{i}^{n-1}+\cdots+a_{n}=0$ for all $i$.

We say that the roots $x_{1}, x_{2}, \ldots, x_{n}$ are in generic position if all Vandermonde matrices $V\left(x_{i_{1}}, x_{i_{2}}, \ldots, x_{i_{k}}\right)=\left(x_{i_{l}}^{m-1}\right), l, m=1,2, \ldots, k$ are invertible over $R$ when $k \geq 2$ and all $i_{1}, i_{2}, \ldots, i_{k}$ are distinct and every entry of such an inverse matrix is invertible.

In this case, one can define the Vandermonde quasideterminant (for the general theory of quasideterminants see [GR, GR2, GGRW])

$$
v\left(x_{i_{1}}, x_{i_{2}}, \ldots, x_{i_{k}}\right)=x_{i_{k}}^{k-1}-r \cdot V\left(x_{i_{1}}, x_{i_{2}}, \ldots, x_{i_{k-1}}\right)^{-1} \cdot c
$$

where $r$ is a row vector of length $k-1, r=\left(x_{i_{1}}^{k-1}, x_{i_{2}}^{k-1}, \ldots, x_{i_{k-1}}^{k-1}\right)$ and $c$ is a column vector of the same length, $c=\left(1, x_{i_{k}}, \ldots, x_{i_{k}}^{k-2}\right)^{T}$.

One can see that $v\left(x_{i_{1}}, x_{i_{2}}, \ldots, x_{i_{k}}\right)$ is an element in the ring $R$. This element is invertible in $R$ because elements $x_{1}, x_{2}, \ldots, x_{n}$ are in a generic position.

Example. $v\left(x_{1}, x_{2}\right)=x_{2}-x_{1}$.

Note that $v\left(x_{i_{1}}, x_{i_{2}}, \ldots, x_{i_{k}}\right)$ is a rational expression in $x_{i_{1}}, x_{i_{2}}, \ldots, x_{i_{k}}$ and that it does not depend of the ordering of $x_{i_{1}}, x_{i_{2}}, \ldots, x_{i_{k-1}}$ (see [GR, GR2, GGRW]).

Set

$$
x_{A, i_{k}}=v\left(x_{i_{1}}, x_{i_{2}}, \ldots, x_{i_{k}}\right) \cdot x_{i_{k}} \cdot v\left(x_{i_{1}}, x_{i_{2}}, \ldots, x_{i_{k}}\right)^{-1},
$$

where $A=\left\{x_{i_{1}}, x_{i_{2}}, \ldots, x_{i_{k-1}}\right\}$. Our notation is justified because $x_{A, i}$ also does not depend on the ordering of $A$. It is convenient to set $x_{\emptyset, i}=x_{i}, i=1, \ldots, n$.

Any ordering $i_{1}, i_{2}, \ldots, i_{n}$ of indices $1,2, \ldots, n$ defines a factorization of $P(t)$. Namely, let $A_{k}=\left\{i_{1}, i_{2}, \ldots, i_{k}\right\}, k=1, \ldots, n-1$ and $A_{0}=\emptyset$. The following result was obtained in [GR2].

\section{Theorem 1.1.1.}

$$
P(t)=\left(t-x_{A_{n-1}, i_{n}}\right)\left(t-x_{A_{n-2}, i_{n-1}}\right) \ldots\left(t-x_{A_{0}, i_{1}}\right) .
$$

The elements $x_{A, i}$ (called pseudo-roots of $P(t)$ in Section 1.2) are defined by pairs $A \subseteq\{1,2, \ldots, n\}$ and $i \in\{1,2, \ldots, n\} \backslash A$. They satisfy the relations 


$$
\begin{gathered}
x_{A \cup\{i\}, j}+x_{A, i}=x_{A \cup\{j\}, i}+x_{A, j} \\
x_{A \cup\{i\}, j} x_{A, i}=x_{A \cup\{j\}, i} x_{A, j}
\end{gathered}
$$

When $A=\emptyset$, the relations (1.1) give relations (0.3).

To understand these relations better, we consider the Hasse graph of the Boolean lattice of subsets of $\{1,2, \ldots, n\}$. Thus, we consider the directed graph whose vertices are subsets of $\{1,2, \ldots, n\}$ and an edge goes from vertex $B$ to vertex $A$ if and only if $B=A \cup\{i\}$ where $i \in\{1,2, \ldots, n\} \backslash A$. Then the relations (1.1) can be described nicely by a diamond in the graph with vertices $A \cup\{i, j\}, A \cup\{i\}, A \cup\{j\}, A$ (see Fig 1 for $A=\emptyset, i=1, j=2$.)

The elements $x_{A \cup\{i\}, j}, x_{A \cup\{j\}, i}, x_{A, j}, x_{A, i}$ correspond to the edges from $A \cup\{i, j\}$ to $A \cup\{i\}$, from $A \cup\{i, j\}$ to $A \cup\{j\}$, from $A \cup\{j\}$ to $A$, and from $A \cup\{i\}$ to $A$.

1.2. Pseudo-roots. The elements $x_{A, i}$ introduced in the previous section are natural examples of pseudo-roots of noncommutative polynomials.

Let $R$ be an associative ring with unit and $P(t)=a_{0} t^{n}+a_{1} t^{n-1}+\cdots+a_{n}$ be a polynomial in $R[t]$. According to [GGRSW] an element $x \in R$ is a pseudo-root of $P(t)$ if there exist polynomials $Q_{1}(t), Q_{2}(t) \in R[t]$ such that

$$
P(t)=Q_{1}(t)(t-x) Q_{2}(t)
$$

The element $x$ is a right root of $P(t)$ if $Q_{2}(t)=1$ and is a left root of $P(t)$ if $Q_{1}(t)=1$. As we mentioned in the introduction, it is easy to check that $x$ is a right root of $P(t)$ if and only if $a_{0} x^{n}+a_{1} x^{n-1}+\cdots+a_{n}=0$. Similarly, $x$ is a left root if and only if $x^{n} a_{0}+x^{n-1} a_{1}+\cdots+a_{n}=0$.

For a noncommutative ring $R$, a theory of polynomials over $R$ should be based not only on properties of right (left) roots but on pseudo-roots as well (see Section 1.1.) In particular, it is natural to study subrings $R_{P}$ of $R$ generated by pseudo-roots of a given polynomial $P(t) \in R[t]$. We will construct now a universal algebra of pseudo-roots $Q_{n}$ introduced in [GRW]. In certain situations there exists a canonical homomorphism of $Q_{n}$ into $R_{P}$.

1.3. The algebra $Q_{n}$. Let $F$ be a field. The algebra $Q_{n}$ over $F$ is generated by elements $x_{A, i}$ where $A \subseteq\{1,2, \ldots, n\}$ and $i \in\{1,2, \ldots, n\} \backslash A$ satisfying relations (1.1).

Let $R$ be an algebra over a field $F$ and let $P(t)=t^{n}+a_{1} t^{n-1}+\cdots+a_{n}$ be a polynomial over $R$ such that $P(t)$ has $n$ right roots $x_{1}, \ldots, x_{n}$ in a generic position. The subalgebra of of $R$ generated by all pseudo-roots of $P(t)$ is denoted by $R_{P}$. The following theorem was proved in [GRW].

Theorem 1.3.1. There exists a homomorphism

$$
\alpha: Q_{n} \rightarrow R_{P}
$$


such that $\alpha\left(z_{A, i}\right)=x_{A, i}$.

It turns out that the algebra $Q_{n}$ has several nice properties studied in [GRW, GGR, GGRSW, SW, Pi]. It is easy to see that this algebra has a set of generators satisfying quadratic relations because linear and quadratic relations in (1.1) are separated, i.e. $Q_{n}$ is a quadratic algebra. In [GRW] we constructed a linear basis in $Q_{n}$, in [GGRSW] we computed the Hilbert series for $Q_{n}$ and its quadratic dual, and in [SW] we showed that $Q_{n}$ is a Koszul algebra.

Other properties of $Q_{n}$, including its relations to the algebra of noncommutative symmetric functions introduced in [GKLLRT], were discussed in [GRW]. Overall, one can say that $Q_{n}$ is a rather "tame" algebra (due to the properties listed above) despite its exponential growth.

\section{The Algebras $\mathbf{A}(i, \Gamma)$ And $\mathbf{A}(\Gamma)$}

2.1. Directed graphs. Let $\Gamma=(V, E)$ be a directed graph. That is, $V$ is a set (of vertices), $E$ is a set (of edges), and $\mathbf{t}: E \rightarrow V$ and $\mathbf{h}: E \rightarrow V$ are functions. (t $(e)$ is the tail of $e$ and $\mathbf{h}(e)$ is the head of $e$.)

A vertex $u \in V$ is called maximal if there is no $e \in E$ such that $\mathbf{h}(e)=u$. A vertex $v \in V$ is called minimal if there is no $e \in E$ such that $\mathbf{t}(e)=v$.

We say that $\Gamma$ is layered if $V$ is the disjoint union of $V_{i}, 0 \leq i \leq n, E$ is the disjoint union of $E_{i}, 0 \leq i \leq n, \mathbf{t}: E_{i} \rightarrow V_{i}, \mathbf{h}: E_{i} \rightarrow V_{i-1}$. We will write $|v|=i$ if $v \in V_{i}$. In this case the number $i$ is called the level of $v$. Note that a layered graph has no loops.

We will assume throughout the remainder of the paper that $\Gamma=(V, E)$ is a layered graph with $V=\cup_{i=0}^{n} V_{i}$, and $V_{0}=\{*\}$ where $*$ is the unique minimal vertex of $\Gamma$.

To any partially ordered set $I$ there corresponds the directed graph $\Gamma_{I}$ called the Hasse graph of $I$. Its vertices are elements $x \in I$ and its edges are pairs $e=(x, y) \in I \times I$ such that $y$ is an immediate predecessor of $x$ (in other words, $y<x$ and there is no $z \in I$ such that $y<z<x$.) The element $x$ is the tail of $e$ and the element $y$ is the head of $e$.

Recall that a partially ordered set $I$ with a function $r: I \rightarrow \mathbb{Z}_{+}$is called a ranked poset with the ranking function if $r(x)>r(y)$ for any $x>y$. Then $r$ turns the corresponding Hasse graph $\Gamma_{I}$ into a layered graph with $|x|=r(x)$. To any maximal (minimal) element in $I$ there corresponds a maximal (minimal) vertex in $\Gamma_{I}$.

Note that every layered graph with no multiple edges arrives in in this manner. Although, the theory we develope below applies to graphs with multiple edges (see Example 1 of Section 2.3), all the examples we consider will be Hasse graphs.

Here are some examples of ranked partially ordered sets.

\section{Examples.}

1. To any set $S$ corresponds the partially ordered set $\mathcal{P}(S)$ of all subsets of $S$. The order relation is given by inclusion and $r(A)$ equals the cardinality of $A$. The minimal 
element in $\mathcal{P}(S)$ is the empty set and the maximal element is $S$. We will always assume that $S$ is a finite set.

2. To any finite-dimensional vector space $E$ over a field $F$ corresponds the partially ordered set $\mathcal{W}(E)$ of all vector subspaces of $E$. The order relation is given by inclusion and $r(W)$ is the dimension of $W$ for a subspace $W \subseteq E$.

The minimal element in $\mathcal{W}(E)$ is the zero subspace $(0)$ and the maximal element is $E$.

3. We say that a layered graph $\Gamma=(V, E)$ with $V=\cup_{i=0}^{n} V_{i}$ is complete if for every $i, 1 \leq i \leq n$, and every $v \in V_{i}, w \in V_{i-1}$, there is a unique edge $e$ with $\mathbf{t}(e)=v, \mathbf{h}(e)=w$. A complete layered graph is determined (up to isomorphism) by the cardinalities of the $V_{i}$. We denote the complete layered graph with $V=\cup_{i=0}^{n} V_{i},\left|V_{i}\right|=m_{i}$ for $0 \leq i \leq n$, by $\mathbf{C}\left[m_{n}, m_{n-1}, \ldots, m_{1}, m_{0}\right]$. Note that the graph $\mathbf{C}\left[m_{n}, m_{n-1}, \ldots, m_{1}, 1\right]$ has a unique minimal vertex of level 0 and the graph $\mathbf{C}\left[1, m_{n-1}, \ldots, m_{1}, m_{0}\right]$ has a unique maximal vertex of level $n$.

4. Recall that the partially ordered set $\mathcal{Y}$ of Young diagrams can be identified with the set of weakly decreasing sequences $\lambda=\left(\lambda_{k}\right)_{k \geq 1}$ such that $\lambda_{k}=0$ for $k>>0$. By definition, $\left(\lambda_{k}\right) \geq\left(\mu_{k}\right)$ if and only if $\lambda_{k} \geq \mu_{k}$ for all $k$. In fact, $\mathcal{Y}$ is a ranked partially ordered set. The rank is defined as $r\left(\left(\lambda_{k}\right)\right)=\sum_{k \geq 1} \lambda_{k}$.

5. Abstract regular polytopes (see, for example, $[\mathrm{MS}]$ ) also are natural examples of ranked partially ordered sets.

6. A family $\mathcal{F} \subseteq \mathcal{P}(S)$ is called a complex if $B \in \mathcal{F}$ and $A \subseteq B$ imply $A \in \mathcal{F}$. The order and the ranking function on $\mathcal{P}(S)$ induce an order and a ranking function on $\mathcal{F}$.

Another important example of a layered graph is the graph of right divisors of a monic polynomial described in the next subsection.

2.2. The graph of right divisors. Let $P(t)$ be a monic polynomial over an associative algebra $R$ and $S$ be a set of pseudo-roots of $P(t)$. Denote by $R_{S}$ the subalgebra in $R$ generated by pseudo-roots $x \in S$.

Construct a layered graph $\Gamma(P, S)=(V, E)$,

$$
V=V_{n} \cup V_{n-1} \cup \ldots V_{1} \cup V_{0}
$$

as follows. The vertices of $V_{k}=\{v \in V: r(v)=k\}$ are monic polynomials $B(t) \in R[t]$ such that $\operatorname{deg} B(t)=k$ and

$$
P(t)=Q(t) B(t)
$$

in $R[t]$.

We say that there is an edge from vertex $B_{1}(t)$ to $B_{2}(t)$ in $\Gamma$ if

$$
B_{1}(t)=(t-x) B_{2}(t)
$$

for some $x \in S$.

Note that graph $\Gamma(P, S)$ has only one maximal vertex $v=P(t)$ and only one minimal vertex $w=1$. 


\subsection{Algebras $\mathbf{A}(i, \Gamma)$ and $\mathbf{A}(\Gamma)$.}

In this section we discuss a class of algebras introduced in [GRSW] and [RSW]. Let $\Gamma=(V, E)$ be a layered directed graph with a finite number of layers. That is, $V=\cup_{i=0}^{n} V_{i}$, $E=\cup_{i=1}^{n} E_{i}, \mathbf{t}: E_{i} \rightarrow V_{i}, \mathbf{h}: E_{i} \rightarrow V_{i-1}$.

Recall that we are assuming throughout the remainder of the paper that $V_{0}=\{*\}$ where $*$ is the unique minimal vertex of $\Gamma$.

For each $v \in \cup_{i=1}^{n} V_{i}$ we will fix, arbitrarily, some $e_{v} \in E$, with $\mathbf{t}(e)=v$. Recall, that if $v \in V_{i}$ we write $|v|=i$ and say that $v$ has level $i$. Similarly, if $e \in E_{i}$ we write $|e|=i$ and say that $e$ has level $i$.

If $v, w \in V$, a path from $v$ to $w$ is a sequence of edges $\pi=\left\{e_{1}, e_{2}, \ldots, e_{k}\right\}$ with $\mathbf{t}\left(e_{1}\right)=v$, $\mathbf{h}\left(e_{k}\right)=w$ and $\mathbf{t}\left(e_{i+1}\right)=\mathbf{h}\left(e_{i}\right)$ for $1 \leq i<k$. We write $v=\mathbf{t}(\pi), w=\mathbf{h}(\pi)$ and call $v$ the tail of the path and $w$ the head of the path. We also write $v>w$ if there is a path from $v$ to $w$.

With $\pi$ defined as above, let $l(\pi)=k$ be the length of $\pi$ and let $|\pi|=\left|e_{1}\right|+\ldots+\left|e_{k}\right|$ be the level of $\pi$.

If $\pi_{1}=\left\{e_{1}, \ldots, e_{k}\right\}, \pi_{2}=\left\{f_{1}, \ldots, f_{l}\right\}$ are paths with $\mathbf{h}\left(\pi_{1}\right)=\mathbf{t}\left(\pi_{2}\right)$ then $\left\{e_{1}, \ldots, e_{k}, f_{1}, \ldots, f_{l}\right\}$ is a path; we denote it by $\pi_{1} \pi_{2}$.

For $v \in V$, write $v^{(0)}=v$ and define $v^{(i+1)}=\mathbf{h}\left(e_{v^{(i)}}\right)$ for $0 \leq i<|v|$. Then $v^{(|v|)}=*$ and $\pi_{v}=\left\{e_{v^{(0)}}, \ldots, e_{v(|v|-1)}\right\}$ is a path from $v$ to $*$.

Let $T(E)$ denote the free associative algebra on $E$ over a field $F$. We are going to introduce a quotient algebra of $T(E)$ modulo relations generalizing relations (0.2) and (0.3). We will do this by equating coefficients of polynomials associated with pairs of paths with the same origin and the same end.

For a path $\pi=\left\{e_{1}, e_{2}, \ldots, e_{m}\right\}$ define

$$
P_{\pi}(\tau)=\left(1-\tau e_{1}\right) \ldots\left(1-\tau e_{m}\right) \in T(E)[\tau] /\left(\tau^{n+1}\right) .
$$

Note that $P_{\pi_{1} \pi_{2}}(\tau)=P_{\pi_{1}}(\tau) P_{\pi_{2}}(\tau)$ if $\mathbf{h}\left(\pi_{1}\right)=\mathbf{t}\left(\pi_{2}\right)$. Write

$$
P_{\pi}(\tau)=\sum_{j=0}^{n+1}(-1)^{j} e(\pi, j) \tau^{j} .
$$

Definition 2.3.1. Let $R$ be the ideal in $T(E)$ generated by

$$
\left\{e\left(\pi_{1}, k\right)-e\left(\pi_{2}, k\right) \mid k \geq 1, \mathbf{t}\left(\pi_{1}\right)=\mathbf{t}\left(\pi_{2}\right), \mathbf{h}\left(\pi_{1}\right)=\mathbf{h}\left(\pi_{2}\right)\right\}
$$

Set

$$
A(\Gamma)=T(E) / R .
$$


In other words, $A(\Gamma)$ is defined by generators $e \in E$ and relations

$$
\begin{gathered}
\sum_{j=1}^{k} e_{j}=\sum_{j=1}^{k} f_{j}, \\
\sum_{i<j} e_{i} e_{j}=\sum_{i<j} f_{i} f_{j}, \\
\ldots \\
e_{1} e_{2} \ldots e_{k}=f_{1} f_{2} \ldots f_{k} .
\end{gathered}
$$

Examples. 1. Suppose $\Gamma=(V, E), e, f \in E$ and $\mathbf{t}(e)=\mathbf{t}(f), \mathbf{h}(e)=\mathbf{h}(f)$. Then the images of $e$ and $f$ in $A(\Gamma)$ are equal. Thus $A(\Gamma)$ is isomorphic to $A\left(\Gamma^{\prime}\right)$ where $\Gamma^{\prime}$ is the graph without multiple edges obtained from $\Gamma$ by identifying all edges with the same tail and head.

2. If $\Gamma$ is a tree-like graph, i.e. there are no distinct paths $\pi_{1}, \pi_{2}$ such that $\mathbf{t}\left(\pi_{1}\right)=\mathbf{t}\left(\pi_{2}\right)$, $\mathbf{h}\left(\pi_{1}\right)=\mathbf{h}\left(\pi_{2}\right)$, then $A(\Gamma)=T(E)$ is the free associative algebra generated by $e \in E$.

3. Let $\Gamma$ be a diamond graph from Fig 1 . Then $A(\Gamma)$ is defined by the relations

$$
\begin{gathered}
e_{1}+e_{2}=f_{1}+f_{2}, \\
e_{1} e_{2}=f_{1} f_{2}
\end{gathered}
$$

and $A(\Gamma)$ is isomorphic to the algebra $Q_{2}$ defined by the relations (1.1).

It also useful to consider larger algebras $A(i, \Gamma)$ associated to directed graphs. To introduce these classes of algebras, define $P_{i, \pi}(\tau)$ to be the image of $P_{\pi}(\tau)$ in the quotient of $T(E)[\tau] /\left(\tau^{i+1}\right)$ and write

$$
P_{i, \pi}(\tau)=\sum_{k=0}^{\min (l(\pi), i)}(-1)^{k} e(i, \pi, k) \tau^{k}
$$

for $i \geq 1$.

Note that $P_{i, \pi_{1} \pi_{2}}(\tau)=P_{i, \pi_{1}}(\tau) P_{i, \pi_{2}}(\tau)$ if $\mathbf{h}\left(\pi_{1}\right)=\mathbf{t}\left(\pi_{2}\right)$.

Set $e(i, \pi, k)=0$ if $k>\min (l(\pi), i)$. For $v \in \cup_{l=1}^{n} V_{l}$, set $P_{i, v}(t)=P_{i, \pi_{v}}(t)$ and $e(i, v, k)=e\left(i, \pi_{v}, k\right)$. Also, set $P_{i, *}(t)=1$ and $e(i, *, k)=0$ if $k>0$.

Definition 2.3.2. Let $R(i)$ be the ideal in $T(E)$ generated by

$$
\left\{e\left(i, \pi_{1}, k\right)-e\left(i, \pi_{2}, k\right) \mid \mathbf{t}\left(\pi_{1}\right)=\mathbf{t}\left(\pi_{2}\right), \mathbf{h}\left(\pi_{1}\right)=\mathbf{h}\left(\pi_{2}\right), 1 \leq k \leq \min \left(l\left(\pi_{1}\right), i\right)\right\} .
$$

Note that

$$
R(1) \subseteq R(2) \subseteq \ldots \subseteq R(n)=R(n+1)=\ldots
$$


Let

$$
A(i, \Gamma)=T(E) / R(i)
$$

Therefore we have

$$
A(1, \Gamma) \rightarrow A(2, \Gamma) \rightarrow \ldots \rightarrow A(n-1, \Gamma) \rightarrow A(n, \Gamma)
$$

Note that $A(n, \Gamma)=A(\Gamma)$ (as in Definition 2.3.1).

In other words, $A(i, \Gamma)$ is defined by generators $e \in E$ and relations

$$
\sum_{\substack{j_{1}<j_{2}<\cdots<j_{k} \\ 1 \leq k \leq i}}\left(e_{j_{1}} e_{j_{2}} \ldots e_{j_{k}}-f_{j_{1}} f_{j_{2}} \ldots f_{j_{k}}\right)
$$

for all pairs of paths $\pi_{1}=\left(e_{1}, e_{2}, \ldots e_{s}\right), \pi_{2}=\left(f_{1}, f_{2}, \ldots f_{s}\right)$ such that $\mathbf{t}\left(\pi_{1}\right)=\mathbf{t}\left(\pi_{2}\right)$, $\mathbf{h}\left(\pi_{1}\right)=\mathbf{h}\left(\pi_{2}\right)$.

In Section 3.3 we will show that for most of the graphs described in Section 2.1, algebras $A(2, \Gamma)$ and $A(\Gamma)$ coincide. In this case $A(\Gamma)$ is described by linear and quadratic relations. In particular, for the Hasse graph $\Gamma_{n}$ of subsets of $\{1,2, \ldots, n\}$ the algebra $A\left(\Gamma_{n}\right)$ is isomorphic to the algebra $Q_{n}$ described in Section 1.3.

2.4. Universality of $A(\Gamma)$. In notations of Section 2.3 assume that graph $\Gamma$ has a unique maximal vertex $M$ and a unique minimal vertex $*$. Define a polynomial $\mathcal{P}_{\Gamma}(t)$ over algebra $A(\Gamma)$ corresponding to any path $\pi_{0}=\left(e_{1}, e_{2}, \ldots, e_{n}\right)$ from $M$ to $*$ :

$$
\mathcal{P}_{\Gamma}(t)=\left(t-e_{1}\right)\left(t-e_{2}\right) \ldots\left(t-e_{n}\right)
$$

It follows from the the definition of $A(\Gamma)$ that $\mathcal{P}_{\Gamma}(t)$ does not depend of a choice of path $\pi_{0}$.

The polynomial $\mathcal{P}_{\Gamma}(t)$ is a monic polynomial. We call it the universal polynomial over $A(\Gamma)$.

Let $R$ be an algebra, $P(t)$ a monic polynomial of degree $n$ over $R$, and $S$ a set of pseudo-roots of $P(t)$. Let $\Gamma(P, S)$ be the layered graph constructed in Section 2.2.

Assume that the set $S$ contains pseudo-roots $a_{1}, a_{2}, \ldots, a_{n}$ such that

$$
P(t)=\left(t-a_{1}\right)\left(t-a_{2}\right) \ldots\left(t-a_{n}\right)
$$

Then graph $\Gamma(P, S)$ contains a directed path from maximal vertex $M=P(t)$ to minimal vertex $*=1$.

Following Section 2.3 construct the algebra $A(\Gamma(P, S))$. Let $\mathcal{P}(t)=P_{\Gamma}(t)$ be the universal polynomial over this algebra. 
Theorem 2.4.1. There is a canonical homomorphism

$$
\kappa: A(\Gamma(P, S)) \rightarrow R
$$

such that the induced homomorphism of polynomial algebras

$$
\hat{\kappa}: A(\Gamma(P, S))[t] \rightarrow R[t]
$$

maps $\mathcal{P}(t)$ to $P(t)$.

Theorem 2.4.1 was proved in [GGRW1]. To construct the homomorphism $\kappa$, note that to any edge $e \in \Gamma(P, S)$ corresponds a pair of polynomials $B_{1}(t), B_{2}(t)$ in $R[t]$ such that $B_{1}(t), B_{2}(t)$ divide $P(t)$ from the right and $B_{1}(t)=(t-a) b_{2}(t)$. Set $\kappa(e)=a$. One can see that $\kappa$ can be uniquely extended to the homomorphism $A(\Gamma(P, S)) \rightarrow R$ and that $\hat{\kappa}(\mathcal{P})(t)=P(t)$.

\section{Properties of the algebras $A(i, \Gamma)$ and $A(\Gamma)$}

Throughout this section that we continue to assume $\Gamma=(V, E)$ is a layered graph with $V=\cup_{i=0}^{n} V_{i}$, that $V_{0}=\{*\}$, and that, for every $v \in V_{+}=\cup_{i=1}^{n} V_{i},\{e \in E \mid \mathbf{t}(e)=v\} \neq \emptyset$. For each $v \in V_{+}$fix, arbitrarily, some $e_{v} \in E$ with $\mathbf{t}\left(e_{v}\right)=v$. This defines a distinguished path, denoted by $\pi_{v}$, from $v$ to $*$. Namely, for $v \in V^{+}$we define $v^{(0)}=v$ and $v^{(i+1)}=$ $\mathbf{h}\left(e_{v^{(i)}}\right)$ for $0 \leq i<|v|$ and we set $\pi_{v}=\left\{e_{v^{(0)}}, e_{v^{(1)}}, \ldots, e_{v^{(|v|-1)}}\right\}$.

3.1. Linear basis in $A(\Gamma)$. For $v \in V_{+}$and $1 \leq k \leq|v|$ we define $\hat{e}(v, k)$ to be the image in $A(\Gamma)$ of the product $e_{1} \ldots e_{k}$ in $T(E)$ where $\pi_{v}=\left\{e_{1}, \ldots, e_{|v|}\right\}$.

If $(v, k),(u, \ell) \in V \times \mathbb{N}$ we say $(v, k)$ covers $(u, \ell)$ if $v>u$ and $k=|v|-|u|$. In this case we write $(v, k)>(u, \ell)$. (In [GRSW] we used different terminology and notation: if $(v, k)>(u, \ell)$ we said $(v, k)$ can be composed with $(u, \ell)$ and wrote $(v, k) \models(u, \ell)$.)

Example. In Fig 1 pair $(\{12\}, 1)$ covers the pairs $(\{1\}, 1)$ and $(\{2\}, 1)$.

The following theorem is proved in [GRSW] (see Corollary 4.5).

Theorem 3.1.1. Let $\Gamma=(V, E)$ be a layered graph, $V=\cup_{i=0}^{n} V_{i}$, and $V_{0}=\{*\}$ where $*$ is the unique minimal vertex of $\Gamma$. Then

$$
\left\{\hat{e}\left(v_{1}, k_{1}\right) \ldots \hat{e}\left(v_{l}, k_{l}\right)\left|l \geq 0, v_{1}, \ldots, v_{l} \in V_{+}, 1 \leq k_{i} \leq\right| v_{i} \mid,\left(v_{i}, k_{i}\right) \ngtr\left(v_{i+1}, k_{i+1}\right)\right\}
$$

is a basis for $A(\Gamma)$.

Theorem 3.1.1 implies the construction of a linear basis in the algebra $Q_{n}$ obtained in [RSW], but the current description is much nicer and the proof is much shorter. 
3.2. New description of the algebras $A(i, \Gamma)$ and $A(\Gamma)$. In Section 2.3 we described algebras $A(i, \Gamma)$ as algebras whose generators are edges of $\Gamma$ subject to homogeneous relations of degree $1,2, \ldots, i$. In this Section, following [RSW], we describe those algebras as algebras whose generators are vertices of $\Gamma$ subject to homogeneous relations of degree $2, \ldots, i$. In fact, we will represent each vertex $v$ in $\Gamma$ by a path from $v$ to $*$. By Definition 2.3.1 the sum of edges in each of such paths has the same image in $A(\Gamma)$.

Define $e(v, 1)=e_{v^{(0)}}+e_{v^{(1)}}+\ldots+e_{v^{(|v|-1)}}$. Thus

$$
e_{v}=e(v, 1)-e\left(v^{(1)}, 1\right)=e\left(\mathbf{t}\left(e_{v}\right), 1\right)-e\left(\mathbf{h}\left(e_{v}\right), 1\right) .
$$

Let $E^{\prime}=\left\{e_{v} \mid v \in V^{+}\right\}$. Let $F$ be our ground field. For any set $X$ denote by $F X$ the vector space over $F$ with basis elements $x \in X$. Define $\tau: F E \rightarrow F E^{\prime}$ by

$$
\tau(f)=e(\mathbf{t}(f), 1)-e(\mathbf{h}(t), 1) .
$$

Then $\tau$ is a projection of $F E$ onto $F E^{\prime}$ with kernel generated by $e\left(i, \pi_{1}, 1\right)-e\left(i, \pi_{2}, 1\right)$ where $\pi_{1}, \pi_{2}$ are paths with the same tail and head.

Now define $\eta: F E^{\prime} \rightarrow F V^{+}$by

$$
\eta: e_{v} \mapsto v
$$

Then $\eta$ is an isomorphism of vector spaces and $\eta \tau$ induces a surjective homomorphism of graded algebras

$$
\theta: T(E) \rightarrow T\left(V^{+}\right) .
$$

Proposition 3.2.1. $\theta$ induces an isomorphism $A(i, \Gamma) \cong T\left(V^{+}\right) / \theta(R(i))$.

It is important to write generators for the ideal $\theta(R(i))$ explicitly. The ideal is generated by elements of the form $\theta\left(e\left(i, \pi_{1}, k\right)-e\left(i, \pi_{2}, k\right)\right)$ where $2 \leq k \leq i$ (see Section 2.3 for the definition of $\left.e\left(i, \pi_{1}, k\right)\right)$. Therefore it will be sufficient to write a formula for $\theta(e(i, \pi, k))$. Let $\pi=\left\{e_{1}, e_{2}, \ldots, e_{m}\right\}$ be a path, let $\mathbf{t}\left(e_{j}\right)=v_{j-1}$ for $1 \leq j \leq m$ and let $\mathbf{h}\left(e_{m}\right)=v_{m}$.

Proposition 3.2.2.

$$
\theta(e(i, \pi, k))=(-1)^{k} \sum_{1 \leq j_{1}<\ldots<j_{k} \leq s}\left(v_{j_{1}-1}-v_{j_{1}}\right) \ldots\left(v_{j_{k}-1}-v_{j_{k}}\right)
$$

where $s=\min (i, m)$.

Proposition 3.2.1 immediately implies

Corollary 3.2.3. $A(2, \Gamma)$ is a quadratic algebra.

We will show below that for many interesting graphs algebra $A(2, \Gamma)$ coincides with algebra $A(\Gamma)$. 


\subsection{Algebras associated with uniform graphs.}

Let $\Gamma$ be a layered graph. For $v \in V$ define $\mathcal{S}_{-}(v)$ to be the set of all vertices $w \in V$ covered by $v$, i.e. such that there exists an edge with the tail $v$ and the head $w$. Similarly, define $\mathcal{S}_{+}(v)$ to be the set of all vertices covering $v$.

For $v \in V_{j}, j \geq 2$, let $\sim_{v}$ denote the equivalence relation on $\mathcal{S}_{-}(v)$ generated by $u \sim_{v} w$ if $\mathcal{S}_{-}(u) \cap \mathcal{S}_{-}(w) \neq \emptyset$.

Definition 3.3.1. The layered graph $V$ is said to be uniform if, for every $v \in V_{j}, j \geq 2$, all elements of $\mathcal{S}_{-}(v)$ are equivalent under $\sim_{v}$.

Example. The diamond graph in Fig 1 is a uniform graph.

Proposition 3.3.2. The Hasse graphs of partially ordered sets listed in Examples 1-5 in Section 2.1 are uniform.

Algebras associated to uniform graphs have especially nice structure.

Proposition 3.3.3. Let $\Gamma$ be a uniform layered graph. Then $A(\Gamma)=A(2, \Gamma) \cong T\left(V^{+}\right) / R_{V}$ is a quadratic algebra and $R_{V}$ is generated by

$$
\left\{v(u-w)-u^{2}+w^{2}+(u-w) x \mid v \in \cup_{i=2}^{n} V_{i}, u, w \in \mathcal{S}_{-}(v), x \in \mathcal{S}_{-}(u) \cap \mathcal{S}_{-}(w)\right\} .
$$

Corollary 3.3.4. The algebra associated to the Hasse graph of all subsets of $\{1,2, \ldots, n\}$ is isomorphic to algebra $Q_{n}$ described in Section 1.3

3.4. $A(\Gamma)$ is a Koszul algebra. Koszul algebras constitute an important class of quadratic algebras (see, for example, [PP, U] for the definitions and properties of Koszul algebras). Most of the known examples are algebras of a polynomial growth. A wide variety of Koszul algebras of exponential growth is given by the following theorem proved in $[\mathrm{RSW}]$.

Theorem 3.4.1. Let $\Gamma$ be a uniform layered graph with a unique minimal element. Then $A(\Gamma)$ is a Koszul algebra.

3.5. Algebras dual to $A(2, \Gamma)$.

Let $A$ be a quadratic algebra over a field $F$. Thus $A$ is isomorphic to a quotient algebra $T(W) /\langle L\rangle$ where $W$ is a vector space over $F$ and $L$ is a subspace in $W \otimes W$. Assume that $W$ is finite dimensional. Denote by $W^{*}$ the dual vector space and set $L^{\perp}=\{f \in$ $\left.W^{*} \otimes W^{*}|f|_{L}=0\right\}$. By definition, the algebra $A^{!}=T\left(W^{*}\right) /<L^{\perp}>$ is the quadratic dual to algebra $A$.

Both algebras $A$ and $A^{!}$are graded and their Hilbert series $H(A, \tau)$ and $H\left(A^{!}, \tau\right)$ are well defined. A graded algebra $A$ is Koszul if and only if $A^{!}$is Koszul and in this case the Hilbert series of $A$ and $A^{!}$are related by

$$
H(A, \tau) H\left(A^{!},-\tau\right)=1
$$


(see, for example, [PP, U].)

According to Corollary 3.2.3 the algebra $A(2, \Gamma)$ is quadratic. Denote its quadratic dual algebra by $B(\Gamma)$. If the graph $\Gamma$ is uniform than $A(2, \Gamma)=A(\Gamma)$ and $B(\Gamma)=A(\Gamma)$ !

The following theorem gives a description of $B(\Gamma)$. When $\Gamma$ is the Hasse graph of the partially ordered set of subsets of $\{1,2, \ldots, n\}$ such description was essentially given in [GGRSW].

Let $\Gamma=(V, E)$ be a layered graph with a unique minimal vertex. Assume that $V$ is a finite set.

Theorem 3.5.1. The algebra $B(\Gamma)$ is generated by vertices $v \in V$ subject to the following relations:

i) $u v=0$ if $u \neq v$ and there is no edge $e \in E$ such $\mathbf{t}(e)=u, \mathbf{h}(e)=v$;

ii) $v^{2}+v \sum w=0$, where the sum is taken over all $w \in \mathcal{S}_{-}(v)$;

iii) $v^{2}+\left(\sum u\right) v=0$, where the sum is taken over all $u \in \mathcal{S}_{+}(v)$.

In Section 4 we will compute Hilbert series for some algebras $B(\Gamma)$ and see that those Hilbert series are polynomial in $\tau$. It follows that in this case, the algebras $B(\Gamma)$ are finite dimensional and the ideal (of codimension one) generated by $V$ is nilpotent. It is not surprising since Theorem 3.5.1 implies that $v^{3}=0$ for any $v \in V$.

\section{The Hilbert series of $A(\Gamma)$}

In this section we compute the Hilbert series of algebras $A(\Gamma)$ introduced in Section 2.3 and specialize the result for some examples of layered directed graphs. All results formulated in this section were obtained in [RSW1].

\subsection{Main theorem.}

Let $h(\tau)$ denote the Hilbert series $H(A(\Gamma), \tau)$, where $\Gamma=(V, E)$ is a layered graph with unique minimal element $*$ of level 0 . Arrange the elements of $V$ in nonincreasing order and index the elements of vectors and matrices by this ordered set. Let 1 denote the column vector all of whose entries are 1, and let $\zeta(\tau)$ denote the matrix with entries $\zeta_{v, w}(\tau)$ for $v, w \in V$ where $\zeta_{v, w}(\tau)=\tau^{|v|-|w|}$ if $v \geq w$ and 0 otherwise. Then we have

\section{Proposition 4.1.1.}

$$
h(\tau)=\frac{1-\tau}{1-\tau \mathbf{1}^{T} \zeta(\tau)^{-1} \mathbf{1}} .
$$

Note that $\zeta(\tau)=1+N$ where $N$ is strongly upper-triangular. Consequently, the $(v, w)$ entry of $\zeta(\tau)^{-1}$ can be written as

$$
\sum_{v=v_{1}>\cdots>v_{\ell}=w \geq *}(-1)^{\ell+1} \tau^{|v|-|w|}
$$

and we have the following result. 
Theorem 4.1.2. Let $\Gamma$ be a layered graph with unique minimal element $*$ of level 0 and $h(\tau)$ denote the Hilbert series of $A(\Gamma)$. Then

$$
h(\tau)=\frac{1-\tau}{1-\sum_{v_{1}>v_{2} \cdots>v_{\ell} \geq *}(-1)^{\ell} \tau^{\left|v_{1}\right|-\left|v_{\ell}\right|+1}} .
$$

The proof of Proposition 4.1.1 and Theorem 4.1.2 is based on Theorem 3.1.1 describing a linear basis in $A(\Gamma)$.

We remark that the matrices $\zeta(1)$ and $\zeta(1)^{-1}$ are well-known as the zeta-matrix and the Möbius-matrix of $V$ (cf. [R]).

In the remaining part of this section we will use Theorem 4.1.2 to compute the Hilbert series of the algebras $A(\Gamma)$ associated with certain layered graphs.

4.2. The Hilbert series of the algebra associated with the Hasse graph of the lattice of subsets of $\{1, \ldots, n\}$.

Let $\Gamma_{n}$ denote the Hasse graph of the lattice of all subsets of $\{1, \ldots, n\}$. Thus the vertices of $\Gamma_{n}$ are subsets of $\{1, \ldots, n\}$, the order relation $>$ is set inclusion $\supset$, the level $|v|$ of a set $v$ is its cardinality, and the unique minimal vertex $*$ is the empty set $\emptyset$. Then the algebra $A\left(\Gamma_{n}\right)$ is the algebra $Q_{n}$ defined in [GRW]. Theorem 4.1.2 implies the following theorem (from [GGRSW]). The proof obtained in $[\mathrm{RSW}]$ is much shorter and more conceptual than that in [GGRSW].

Theorem 4.2.1.

$$
H\left(Q_{n}, \tau\right)=\frac{1-\tau}{1-\tau(2-\tau)^{n}}
$$

4.3. The Hilbert series of algebras associated with the Hasse graph of the lattice of subspaces of a finite-dimensional vector space over a finite field.

We will denote by $\mathbf{L}(n, q)$ the Hasse graph of the lattice of subspaces of an $n$-dimensional space over the field $\mathbf{F}_{q}$ of $q$ elements. Thus the vertices of $\mathbf{L}(n, q)$ are subspaces of $\mathbf{F}_{q}^{n}$, the order relation $>$ is inclusion of subspaces $\supset$, the level $|U|$ of a subspace $U$ is its dimension, and the unique minimal vertex $*$ is the zero subspace $(0)$. Recall that $\left(\begin{array}{c}n \\ m\end{array}\right)_{q}$ is a $q$-binomial coefficient.

\section{Theorem 4.3.1.}

$$
\frac{1-\tau}{H(A(\mathbf{L}(n, q)), \tau)}=1-\tau \sum_{m=0}^{n}\left(\begin{array}{c}
n \\
m
\end{array}\right)_{q}(1-\tau)(1-\tau q) \ldots\left(1-\tau q^{n-m-1}\right) .
$$

Note that setting $q=1$ in the expression in Theorem 4.3 .1 gives $1-\tau(2-\tau)^{n}$. By Theorem 4.2.1, this is $(1-\tau) H\left(Q_{n}, \tau\right)^{-1}$.

Since by Theorem 3.4.1 (see also [RSW]) $A(\mathbf{L}(n, q)$ ) is a Koszul algebra, we have the following corollary. 


\section{Corollary 4.3.2.}

$$
H\left(A(\mathbf{L}(n, q))^{!}, \tau\right)=1+\sum_{m=0}^{n-1}\left(\begin{array}{l}
n \\
m
\end{array}\right)_{q}(1+\tau q) \ldots\left(1+\tau q^{n-m-1}\right) .
$$

\subsection{The Hilbert series of algebras associated with complete layered graphs.}

Recall (see Example 3, Section 2.1) that a layered graph $\Gamma=(V, E)$ with $V=\cup_{i=0}^{n} V_{i}$ is complete if for every $i, 1 \leq i \leq n$, and every $v \in V_{i}, w \in V_{i-1}$, there is a unique edge $e$ with $\mathbf{t}(e)=v, \mathbf{h}(e)=w$. A complete layered graph is determined (up to isomorphism) by the cardinalities of the $V_{i}$. We denote the complete layered graph with $V=\cup_{i=0}^{n} V_{i},\left|V_{i}\right|=m_{i}$ for $0 \leq i \leq n$, by $\mathbf{C}\left[m_{n}, m_{n-1}, \ldots, m_{1}, m_{0}\right]$. Note that the graph $\mathbf{C}\left[m_{n}, m_{n-1}, \ldots, m_{1}, 1\right]$ has a unique minimal vertex of level 0 and so Theorem 4.1 .2 applies to $A\left(\mathbf{C}\left[m_{n}, m_{n-1}, \ldots, m_{1}, 1\right]\right)$. This leads to the following theorem.

\section{Theorem 4.4.1.}

$$
\begin{gathered}
\frac{1-\tau}{H\left(A\left(\mathbf{C}\left[m_{n}, m_{n-1}, \ldots, m_{1}, 1\right], \tau\right)\right.}= \\
1-\sum_{k=0}^{n} \sum_{a=k}^{n}(-1)^{k} m_{a}\left(m_{a-1}-1\right)\left(m_{a-2}-1\right) \ldots\left(m_{a-k+1}-1\right) m_{a-k} \tau^{k+1} .
\end{gathered}
$$

This result applies, in particular, to the case $m_{0}=m_{1}=\ldots=m_{n}=1$. The resulting algebra $A(\mathbf{C}[1, \ldots, 1])$ has $n$ generators and no relations. Theorem 4.4 .1 shows that

$$
\frac{1-\tau}{H(A(\mathbf{C}[1, \ldots, 1]), \tau)}=1-\sum_{a=0}^{n} \tau+\sum_{a=1}^{n} \tau^{2}=(1-\tau)(1-n \tau)
$$

Thus

$$
H(A(\mathbf{C}[1, \ldots, 1]), \tau)=\frac{1}{1-n \tau}
$$

and we have recovered the well-known expression for the Hilbert series of the free associative algebra on $n$ generators.

Since by Theorem 3.4.1 (see also [RSW]) the algebras associated to complete directed graphs are Koszul algebras, we have the following corollary.

\section{Corollary 4.4.2.}

$$
\begin{gathered}
H\left(A\left(\mathbf{C}\left[m_{n}, m_{n-1}, \ldots, m_{1}, 1\right]\right) !, \tau\right)= \\
1+\sum_{k=1}^{n} \sum_{a=k}^{n} m_{a}\left(m_{a-1}-1\right)\left(m_{a-2}-1\right) \ldots\left(m_{a-k+1}-1\right) \tau^{k} .
\end{gathered}
$$




\section{Sufficient Sets of PSEUdo-Roots And DiRECTEd GRAPHS}

We return to questions 1) and 2) from the introduction: Given a polynomial $P(t)$ over a noncommutative algebra, how to obtain its factorizations and how to relate two different factorizations? To answer these questions we will work in general context of algebras $A(\Gamma)$.

5.1. Defining sets of pseudo-roots. In this section we briefly describe some results from [GGRW1]. Let $R$ be an associative ring with unit, $P(t)=t^{n}+a_{1} t^{n-1}+\cdots+a_{n}$ be a polynomial over $R$, and $t$ be a central variable.

Recall (see Theorem 1.1.1) that if $P(t)$ has right roots $x_{1}, x_{2}, \ldots, x_{n}$ in a generic position then $P(t)$ admits factorizations

$$
P(t)=\left(t-x_{A_{n-1}, i_{n}}\right)\left(t-x_{A_{n-2}, i_{n-1}}\right) \ldots\left(t-x_{\emptyset, i_{1}}\right)
$$

indexed by orderings of $\{1,2, \ldots, n\}$. Also recall that pseudo-roots $x_{A, i}$ 's are rational expressions in $x_{1}, x_{2}, \ldots, x_{n}$.

According to [GRW] the pseudo-roots $x_{A, i}$ 's can be obtained from a generic set of $n$ right roots by a sequence of operations

$$
a, b \mapsto(a-b) a(a-b)^{-1}, \quad(b-a) b(b-a)^{-1}
$$

and from a generic set of $n$ left roots by a sequence of operations

$$
a, b \mapsto(a-b)^{-1} a(a-b), \quad(b-a)^{-1} b(b-a)
$$

This leads us to the following natural question. We call a set of pseudo-roots $Y=$ $\left\{y_{1}, y_{2}, \ldots, y_{n}\right\}$ of $P(t)$ is a defining set if

$$
P(t)=\left(t-y_{n}\right)\left(t-y_{n-1}\right) \ldots\left(t-y_{1}\right)
$$

Our question is then: given a set of pseudo-roots $Z$, when it is possible to construct a defining set of pseudo-roots from elements of $Z$ by a successive application of operations of (5.1)-type?

Our answer to this question is based on a geometrical "diamond" interpretation of operations of (5.1)-type.

\subsection{Sufficient sets of pseudo-roots and the algebra $Q_{n}$.}

To avoid taking inverses, we will slightly change the definition of operations of (5.1)type. Recall that algebra $Q_{n}$ corresponds to the Hasse graph $\Gamma_{n}$ of the Boolean lattice of $\{1,2, \ldots$,$\} and Q_{n}[t]$ contains a unique universal polynomial, denoted $\mathcal{P}(t)$ (as defined in Section 2.4.) 
Definition 5.2.1. We say that a pseudo-root $\xi \in Q_{n}$ is obtained from an ordered pair of pseudo-roots $x_{A, i}, x_{B, j}$ by the u-operation if $A \cup\{i\}=B \cup\{j\}$ and $\left(x_{A, i}-x_{B, j}\right) x_{A, i}=$ $\xi\left(x_{A, i}-x_{B, j}\right)$.

A pseudo-root $\eta \in Q_{n}$ is obtained from an ordered pair of pseudo-roots $x_{A, i}, x_{B, j}$ by the $d$-operation if $A \cup\{i\}=B \cup\{j\}$ have and $\left(x_{A, i}-x_{B, j}\right) \eta=x_{A, i}\left(x_{A, i}-x_{B, j}\right)$.

Proposition 5.2.2. The element $x_{A \cup\{i\}, j}$ is obtained by the u-operation from the pair $x_{A, j}, x_{A, i}$.

The element $x_{A, i}$ is obtained by the d-operation from the pair $x_{A \cup\{j\}, i}, x_{A \cup\{i\}, j}$.

Definition 5.2.3. The set of elements in $Q_{n}$ that can be obtained from elements of $Z$ by a successive applications of $d$ - and $u$-operations is called the du-envelope of $Z$.

Definition 5.2.4. A set $Z \subseteq Q_{n}$ is called sufficient if the $d u$-envelope of $Z$ contains a defining set of pseudo-roots of $\mathcal{P}(t)$.

Any defining set of elements is a sufficient set. Other examples of sufficient sets in $Q_{n}$ are given by the following statement.

Proposition 5.2.5. The sets $\left\{x_{\emptyset, k} \mid 1 \leq k \leq n\right\}$ and $\left\{x_{\{12 \ldots \hat{k} \ldots n\}, k} \mid 1 \leq k \leq n\right\}$ are sufficient in $Q_{n}$ for $\mathcal{P}(t)$.

A necessary condition for a subset in $Q_{n}$ to be sufficient for $\mathcal{P}(t)$ is given by the following theorem.

Theorem 5.2.6. If $Z=\left\{x_{A_{1}, i_{1}}, x_{A_{2}, i_{2}}, \ldots, x_{A_{n}, i_{n}}\right\}$ is a sufficient subset of $Q_{n}$ then $i_{1}, i_{2}, \ldots, i_{n}$ are distinct.

A set of edges in a directed graph is connected if it is connected in the associated non-directed graph (see Section 5.3 below for details).

Theorem 5.2.7. Let $Z=\left\{x_{A_{1}, i_{1}}, x_{A_{2}, i_{2}}, \ldots, x_{A_{n}, i_{n}}\right\}$ be a subset of $Q_{n}$ such that $i_{1}, i_{2}$, $\ldots, i_{n}$ are distinct. If the set of edges $\left\{\left(A_{1}, i_{1}\right),\left(A_{2}, i_{2}\right), \ldots,\left(A_{n}, i_{n}\right)\right\}$ in $\Gamma_{n}$ is connected then the set $Z$ is sufficient.

Let $f: Q_{n} \rightarrow D$ be a homomorphism of $Q_{n}$ into a division ring $D, \hat{f}: Q_{n}[t] \rightarrow D[t]$ be the induced homomorphism of the polynomial rings and $P(t)=\hat{f}(\mathcal{P}(t))$.

Corollary 5.2.8. Let $Z=\left\{x_{A_{1}, i_{1}}, x_{A_{2}, i_{2}}, \ldots, x_{A_{n}, i_{n}}\right\}$ be a subset of $Q_{n}$ such that $i_{1}, i_{2}$, $\ldots, i_{n}$ are distinct and the set of edges $\left\{\left(A_{1}, i_{1}\right),\left(A_{2}, i_{2}\right), \ldots,\left(A_{n}, i_{n}\right)\right\}$ in $\Gamma_{n}$ is connected. Then all coefficients of $P(t) \in D[t]$ can be obtained from elements $f(z), z \in Z$, by operations of addition, subtractions, multiplication, and left and right conjugation.

Examples. 1. The set $X=\left\{x_{\emptyset, 1}, x_{\emptyset, 2}, \ldots, x_{\emptyset, n}\right\}$ is connected (the corresponding edges have a common head $\emptyset$ ). Therefore, $X$ is a sufficient set.

2. For $n=2$ the sufficient sets are $\left\{x_{\{i\}, j}, x_{\emptyset, i}\right\},\left\{x_{\emptyset, j}, x_{\emptyset, i}\right\},\left\{x_{\{i\}, j}, x_{\{j\}, i}\right\}$. The sets $\left\{x_{\{i\}, j}, x_{\emptyset, j}\right\}$ are not sufficient. Here $i, j=1,2, i \neq j$. 
3. Let $n=3$. The set $\left\{x_{\{1\}, 2}, x_{\{2\}, 1}, x_{\{1\}, 3}\right\}$ is sufficient because

$$
\begin{gathered}
\left(x_{\{2\}, 1}-x_{\{1\}, 2}\right) x_{\emptyset, 1}=x_{\{1\}, 2}\left(x_{\{2\}, 1}-x_{\{1\}, 2}\right), \\
x_{\{12\}, 3}\left(x_{\{1\}, 3}-x_{\{1\}, 2}\right)=\left(x_{\{1\}, 3}-x_{\{1\}, 2}\right) x_{\{1\}, 3}
\end{gathered}
$$

and $\left\{x_{\{12\}, 3}, x_{\{1\}, 2}, x_{\emptyset, 1}\right\}$ is a defining set of pseudo-roots.

The sets $\left\{x_{\{1\}, 2}, x_{\{2\}, 1}, x_{\emptyset, 3}\right\}$ and $\left\{x_{\{1\}, 3}, x_{\{1\}, 2}, x_{\emptyset, 1}\right\}$ also are sufficient but not defining sets in $Q_{3}$.

4. The set $W=\left\{x_{\{12\}, 3}, x_{\{3\}, 2}, x_{\emptyset, 1}\right\}$ is not sufficient because $d$ - and $u$-operations are not defined on elements of $W$.

Theorem 5.2.7 follows from a more general theorem for algebras associated with directed graphs.

\subsection{Sufficient sets of edges for directed graphs.}

In this section we will define and study sufficient sets of edges in directed graphs $\Gamma=$ $(V, E)$. These sets will provide us with a construction of sufficient sets of pseudo-roots of polynomials $\mathcal{P}(t)$ over algebras $A(\Gamma)$. All graphs considered in this sections are simple (i.e., if $t(e)=t(f)$ and $h(e)=h(f)$ then $e=f$ ) and acyclic (i.e., there are no directed paths $P$ such that $t(P)=h(P))$.

Let $\Gamma=(V, E)$ be a directed graph.

\section{Definition 5.3.1.}

(1) A pair of edges $f_{1}, f_{2}$ with a common head is obtained from the pair $e_{1}, e_{2}$ with a common tail by $D$-operation if $h\left(e_{i}\right)=t\left(f_{i}\right)$ for $i=1,2$;

(2) A pair of edges $e_{1}^{\prime}, e_{2}^{\prime}$ with a common tail is obtained from the pair $f_{1}^{\prime}, f_{2}^{\prime}$ with a common head by $U$-operation if $h\left(e_{i}^{\prime}\right)=t\left(f_{i}^{\prime}\right)$ for $i=1,2$.

Example. In Fig 1 edges $f_{1}, f_{2}$ can be obtained from edges $e_{1}, e_{2}$ by a $D$-operation. Conversely, edges $e_{1}, e_{2}$ can be obtained from edges $f_{1}, f_{2}$ by an $U$-operation.

Remark. We do not require the uniqueness of $D$ - and $U$-operations.

Definition 5.3.2. A subset $E_{0} \subseteq E$ is called $D U$-complete (or simply complete) if the results of any $D$-operation or any $U$-operation applied to edges from $E_{0}$ belong to $E_{0}$.

Proposition 5.3.3. For any subset $F \subseteq E$ there exists a minimal DU-complete set $\hat{F} \subseteq E$ containing $F$.

We call $\hat{F}$ the completion of $F$.

Let $\Gamma=(V, E)$ be a directed graph.

Definition 5.3.4. A set of edges $G$ in $\Gamma$ is called sufficient if its completion $\hat{G}$ contains a path from a maximal vertex (source) to a minimal vertex (sink). 
Definition 5.3.5. A set of vertices $W \subseteq V$ is called ample if

(1) For any non-minimal vertex $v \in V$ there exists a vertex $u \in W$ such that there is no directed path in $\Gamma$ from $u$ to $v$;

(2) For any non-maximal vertex $v \in V$ there exists a vertex $w \in W$ such that there is no directed path in $\Gamma$ from $v$ to $w$.

A set of edges is called ample if the set of its tails and heads is ample.

As an example, consider the graph $\Gamma_{n}$ of all subsets of $\{1, \ldots, n\}$. It has one source $\{1, \ldots, n\}$ and one sink $\emptyset$.

Proposition 5.3.6. A set of edges $\left(A_{1}, i_{1}\right),\left(A_{2}, i_{2}\right), \ldots,\left(A_{n}, i_{n}\right)$ in $\Gamma_{n}$ is ample if $i_{1}, i_{2}$, $\ldots, i_{n}$ are distinct.

Definition 5.3.7. A directed graph is called a modular graph if:

(1) For any two edges $e_{1}, e_{2}$ with a common tail there exist edges $f_{1}, f_{2}$ with a common head such that $h\left(e_{i}\right)=t\left(f_{i}\right)$ for $i=1,2$;

(2) For any two edges $h_{1}, h_{2}$ with a common head there exist edges $g_{1}, g_{2}$ with a common tail such that $h\left(g_{i}\right)=t\left(h_{i}\right)$ for $i=1,2$.

We do not require the uniqueness of $f_{1}, f_{2}$ and $h_{1}, h_{2}$.

Theorem 5.3.8. Any ample connected set of edges of a finite modular directed graph is a sufficient set.

Now let $\Gamma=(V, E)$ be a directed graph such that

(1) $\Gamma$ contains a unique source $M$ and a unique sink $m$;

(2) For each vertex $v \in V$ there exist a directed path from $M$ to $v$ and a directed path from $v$ to $M$.

Recall that we associate to $\Gamma$ an algebra $A(\Gamma)$ and the universal polynomial $\mathcal{P}(t) \in$ $A(\Gamma)[t]$. The polynomial $\mathcal{P}(t)$ is constructed using a path $e_{1}, e_{2}, \ldots, e_{n}$ from $M$ to $m$ in $\Gamma$, but it does not depend on the path. To any edge $e \in E$ there corresponds to a pseudo-root $e \in A(\Gamma)$ of $\mathcal{P}(t)$, and to any path $\left(e_{1}, e_{2}, \ldots, e_{n}\right)$ from $M$ to $m$ in $\Gamma$ there corresponds the factorization

$$
\mathcal{P}(t)=\left(t-e_{1}\right)\left(t-e_{2}\right) \ldots\left(t-e_{n}\right)
$$

of $\mathcal{P}(t)$ over $A(\Gamma)$.

Theorem 5.3.8 implies

Theorem 5.3.9. Let $S \subseteq E$ be an ample connected set of edges in a modular directed graph $\Gamma=(V, E)$. Then there exists a factorization (3.1) of $\mathcal{P}(t)$ such that the DU-completion of $S$ contains elements $e_{1}, e_{2}, \ldots, e_{n}$ and, therefore, coefficients of $\mathcal{P}(t)$. 


\section{REFERENCES}

[B] A. Borodin, Isomonodromy transformations of linear systems of difference equations, Ann. of Math. 160 (2004), 1141-1182.

[GGR] I. Gelfand, S. Gelfand, and V. Retakh, Noncommutative algebras associated to complexes and graphs, Selecta Math. (NS) 7 (2001), 525-531.

[GGRSW] I. Gelfand, S. Gelfand, V. Retakh, S. Serconek, and R. Wilson, Hilbert series of quadratic algebras associated with decompositions of noncommutative polynomials, J. Algebra 254 (2002), 279-299.

[GGRW] I. Gelfand, S. Gelfand, V. Retakh, R. Wilson, Quasideterminants, Advances in Math. 193 (2005), 56-141.

[GGRW1] I. Gelfand, S. Gelfand, V. Retakh, R. Wilson, Factorizations of polynomials over noncommutative algebras and sufficient sets of edges in directed graphs, Lett. Math. Physics 74, 153-167.

[GLR] I. Gohberg, P. Lancaster, and L. Rodman, Matrix polynomials, Academic Press, 1982.

[GR] I. Gelfand, V. Retakh, Determinants of matrices over noncommutative rings, Funct. Anal. Appl. (1991), 91-102.

[GR1] I. Gelfand, V. Retakh, Gelfand Mathematical Seminars 1993-95, Birkhauser Boston, 1996, pp. 93-100.

[GR2] I. Gelfand, V. Retakh, Quasideterminants I, Selecta Math. (N.S.) 3 (1997), 517-546.

[GRSW] I. Gelfand, V. Retakh, S. Serconek, and R. Wilson, On a class of algebras associated to directed graphs, Selecta Math. (N.S.) 11 (2005), 281-295.

[GRW] I. Gelfand, V. Retakh, and R. Wilson, Quadratic-linear algebras associated with decompositions of noncommutative polynomials and Differential polynomials, Selecta Math. (N.S.) 7 (2001), 493-523.

[L] T.Y. Lam, A first course in noncommutative rings, Springer-Verlag, 2001.

[LL] T.Y. Lam and A. Leroy, Wedderburn polynomials over division rings. I., J. Pure Appl. Algebra 186, 43-76.

[MS] P. McMullen, E. Schulte, Abstract Regular Polytopes, Cambridge University Press, 2002.

[N] I. Niven, Equations in quaternions, Amer. Math. Monthly 48, 654-661.

[O] A. Odesskii, Set-theoretical solutions to the Yang-Baxter relation from factorization of matrix polynomials and $\theta$-functions, Mosc. Math. J. 3 (2003), no. 1, 97-103, 259.

[Pi] D. Piontkovski, Algebras associated to pseudo-roots of noncommutative polynomials are Koszul, Int. J. Algebra and Comp. 15 (2005), 643-648.

[PP] A. Polishchuk, and L. Positselski, Quadratic Algebras, American Math. Society, 2005.

[RSW] V. Retakh, S. Serconek, and R. Wilson, On a class of Koszul algebras associated to directed graphs, math.QA/0508303, J. Algebra (to appear).

[RSW1] V. Retakh, S. Serconek, and R. Wilson, Hilbert series of algebras associated to directed graphs, math.CO/0601224, J. Algebra (to appear).

[R] Rota G.-C., On the foundations of combinatorial theory, I. Theory of Möbius functions, Z. Wahrscheinlichkeitstheorie und Verw. Gebiete 2 (1964), 340-368.

[SW] S. Serconek and R. L. Wilson, Quadratic algebras associated with decompositions of noncommutative polynomials are Koszul algebras, J. Algebra 278 (2004), 473-493.

[U] V.A. Ufnarovskij, Algebra, Vol. VI, Springer-Verlag, New York, 1995, pp. 1-196.

[V] A. Veselov, Yang-Baxter maps and integrable dynamics, Phys. Lett. A 314 (2003), no. 3, $214-221$.

[W] R. L. Wilson, Equacoes Polinomiaia Sobre Matrizes, Revista da Olimpiada, Universidade Federale de Goias 5 (2004), 67-85. 
V.R., R.W.: Department of Mathematics, Rutgers University, Piscataway, NJ 08854-8019 S.S: IME-UFG CX Postal 131 Goiania - GO CEP 74001-970 Brazil

$$
\text { E-mail address: }
$$

vretakh@math .rutgers . edu

serconek@math.rutgers.edu

rwilson@math.rutgers.edu 